\title{
Research Article \\ Vehicle Vibration Analysis in Changeable Speeds Solved by Pseudoexcitation Method
}

\author{
Li-Xin Guo and Li-Ping Zhang \\ School of Mechanical Engineering and Automation, Northeastern University, Shenyang 110004, China \\ Correspondence should be addressed to Li-Xin Guo, lxguo@mail.neu.edu.cn
}

Received 31 December 2009; Revised 20 February 2010; Accepted 28 August 2010

Academic Editor: Carlo Cattani

Copyright (c) 2010 L.-X. Guo and L.-P. Zhang. This is an open access article distributed under the Creative Commons Attribution License, which permits unrestricted use, distribution, and reproduction in any medium, provided the original work is properly cited.

The vehicle driving comfort has become one of the important factors of vehicle quality and receives increasing attention. In this paper, the mechanical and mathematical models of the halfcar, five degrees of freedom (DOF) of a vehicle were established, as well as the pseudoexcitation model of road conditions for the front wheel and the rear wheel. By the pseudoexcitation method, the equations of transient response and power spectrum density were established. After numerical simulation to vehicle vibration response of changeable driving, the results show that the pseudoexcitation method is more convenient than the traditional method and effectively solves the smoothness computation problems of vehicles while the pseudoexcitation method is used to analyze vehicle vibration under nonstationary random vibration environments.

\section{Introduction}

The mechanical and mathematical model of vehicle systems is usually simplified as a multiple-mass, complicated vibration system. Due to road excitation, vehicles may come into complicated vibration, which is disadvantageous to passenger health and goods protection $[1,2]$. Therefore, it is important and necessary to control the vehicle's vibration within a limited and comfortable grade in order to ensure safety steering and physical health of drivers and passengers, as well as the operating stability of man-vehicle-road system. In the process of automobile moving, the random and changeable road surface is the main factor to induce vehicle vibration. Therefore, investigation of vehicle's stochastic vibration $[3,4]$ induced by road excitation has been a significant problem of vehicle design and its performance simulation.

At present, for this kind of problems, the Fourier transform analysis is used to investigate the dynamic characteristics of constant driving problems of automobiles based on stationary random vibration theory. After finishing vibration model of vehicles, it is important to derive the frequency characteristic of vehicle vibration responses and to 
establish power spectrum density function of road excitation and vehicle vibration responses [5]. Then it can be used to analyze the influence of vehicle structural parameters and road excitation on vehicle random vibration [6]. Although this method was relatively simple, its derivation process is too complicated, that is, this method needs not only to derive the frequency response characteristics of vehicle vibration system, but also to derive the frequency response characteristics of vehicle vibration response values.

However, in some circumstances, vehicles are running in changeable speeds, such as in accelerating starting period and decelerating stopping period. In these cases, the road excitation and the vehicle dynamic response in time domain are nonstationary $[7,8]$. The stochastic vibration analysis method based on Fourier transform and its inverse transform has been used to study the changeable speed response of vehicles in unevenness roads, but its computation work is enormous. The pseudoexcitation method was used to analyze the stochastic vibration of structural systems [9-11]. By pseudoexcitation method, stochastic vibration analysis was carried on a two-DOF system vibration of a quarter-vehicle model [12$14]$, in which the vibration response of a constant speed moving vehicle was investigated to a stationary random road excitation. The changeable speed vehicle vibration response was also conducted under one-point road excitation [15]. In addition, some last investigations $[16,17]$ have dealt with a quarter-car model with a harmonic excitation while the study [18] considers the additional stochastic component in the road surface roughness. In this study, the time-space frequency relationship of vehicle vibration under changeable speed moving was derived by pseudoexcitation method, and then the equation of transient power spectrum density of vehicle vibration response under nonstationary random road excitation input was obtained. At the end, we also conducted the numerical simulations to the vibration responses of a half-car, five-DOF vehicle system under changeable speed moving conditions.

\section{Theory of Pseudoexcitation Method}

When a linear system is randomly excited by self-spectrum density $S_{x x}(w)$, the self-power spectrum of its response $y$ is

$$
S_{y y}(w)=|H(w)|^{2} S_{x x}(w)
$$

where $H(w)$ is the frequency response function, and its meaning is shown in Figure 1 [19], that is, the corresponding harmonic response is $y=H(w) e^{i w t}$ when the random excitation is replaced by harmonic excitation $e^{i w t}$. From Figure 1, it can be seen that if it multiplies a constant $\sqrt{S_{x x}}$ before the excitation $e^{i w t}$, it can create a pseudoexcitation,

$$
\tilde{x}(t)=\sqrt{S_{x x}} e^{i w t}
$$

After multiplying a same constant to its response value, it can give the following equations:

$$
\begin{gathered}
\tilde{y}^{*} \tilde{y}=|\tilde{y}|^{2} S_{x x}=S_{y y}, \\
\tilde{x}^{*} \tilde{y}=\sqrt{S_{x x}} e^{-i w t} \cdot \sqrt{S_{x x}} e^{i w t}=S_{x x} H=S_{x y}, \\
\tilde{y}^{*} \tilde{x}=\sqrt{S_{x x}} e^{-i w t} \cdot \sqrt{S_{x x}} H e^{i w t}=S_{x x} H=S_{s y} .
\end{gathered}
$$



(a) $S_{x x}$

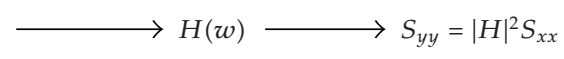
(b) $x=e^{i w t}$

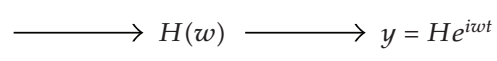
(c) $\tilde{x}=\sqrt{S_{x x}} e^{i w t}$

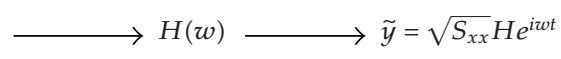
(d) $\tilde{x}=\sqrt{S_{x x}} e^{i w t}$

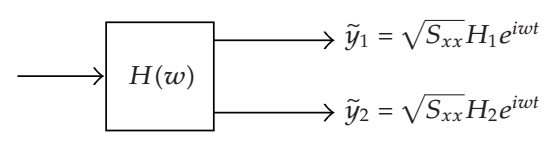

Figure 1: The principle of pseudoexcitation method.

The last formula in the above 3 equations is the conventional expressions of self-spectrum density or mutual spectrum density.

If the pseudoresponse values $\tilde{y}_{1}$ and $\tilde{y}_{2}$ are considered in the above system, the following equations can be validated:

$$
\begin{gathered}
\tilde{y}_{1}^{*} \tilde{y}_{2}=H_{1}{ }^{*} \sqrt{S_{x x}} e^{-i w t} \cdot H_{2} \sqrt{S_{x x}} e^{i w t}=H_{1}{ }^{*} S_{x x} H_{2}=S_{y 1 y 2} \\
\tilde{y}_{2}^{*} \tilde{y}_{1}=H_{2}{ }^{*} S_{x x} H_{1}=S_{y 2 y 1} .
\end{gathered}
$$

Then the matrixes of power spectrum density are as follows:

$$
\begin{aligned}
& {\left[S_{y y}\right]=\{\tilde{y}\}^{*} \cdot\{\tilde{y}\}^{T},} \\
& {\left[S_{x y}\right]=\{\tilde{x}\}^{*} \cdot\{\tilde{y}\}^{T},} \\
& {\left[S_{y x}\right]=\{\tilde{y}\}^{*} \cdot\{\tilde{x}\}^{T} .}
\end{aligned}
$$

If the pseudoexcitation of a random process is $\tilde{x}(t)=\sqrt{S_{x x}} e^{i w t}$, then it can give

$$
\begin{gathered}
\dot{\tilde{x}}=i w \sqrt{S_{x x}} e^{i w t}, \quad \ddot{\tilde{x}}=-w^{2} \sqrt{S_{x x}} e^{i w t}, \\
S_{\ddot{x} \ddot{x}}=w^{4} S_{x x} .
\end{gathered}
$$

That is the power spectrum density of accelerations, and in the same way, we can obtain the following equations:

$$
\begin{aligned}
S_{\ddot{y} \ddot{\tilde{y}}} & =\{\ddot{\tilde{y}}\}^{*} \cdot\{\ddot{\tilde{y}}\}^{T}, \\
S_{\ddot{y}_{1} \ddot{y}_{2}} & =\left\{\ddot{\tilde{y}}_{1}\right\}^{*} \cdot\left\{\ddot{\tilde{y}}_{2}\right\}^{T},
\end{aligned}
$$

where $*$ is a complex conjugate, and $T$ is a matrix transfer. 


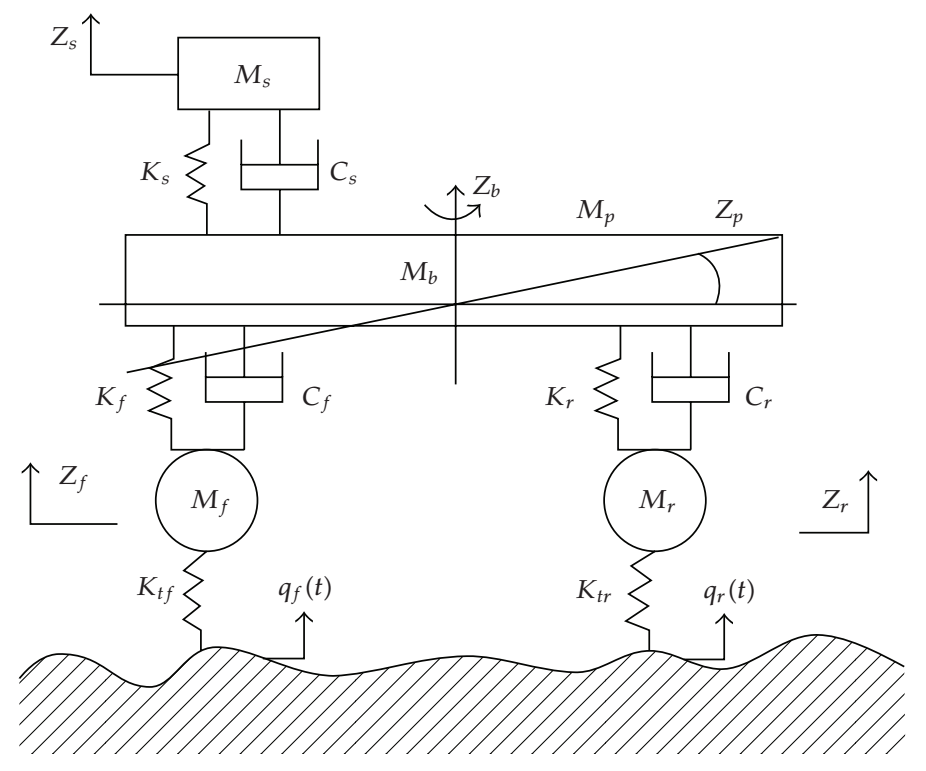

Figure 2: The mechanical model of a half-car, five-DOF automobile system.

After computing the vibration response of the system in the case of the pseudo harmonic excitation, all the power spectrum densities of them can be solved according to (2.5)-(2.9). Then the self-spectrum density and mutual spectrum density of them can also be obtained.

\section{Vibration Analysis of the Half-Car, Five-DOF Vehicle System in Changeable Speeds}

\subsection{Mechanical and Mathematical Models of the System}

For analyzing automobile vibration, it is important to establish its mechanical and mathematical model of the automobile structural system, so that the vibration characteristic response value of the mathematical model of vehicle vibration can be solved and obtained. For the mechanical modeling of automobiles, a seven-DOF mechanical model [20] has been developed to investigate the influence of active and semiactive suspension to automobile dynamic performance. In addition, the moving smartness and operating stability of automobiles were also investigated by spatial mechanical models of automobiles [21]. In this study, a half-car, five-DOF linear mechanical model [22] of an automobile system was developed as shown in Figure 2.

In Figure 2, $m_{s}$ is the mass of driver and chair and $m_{b}$ is the mass of automobile structure. $m_{f}, m_{r}$ are the nonspring supported mass of front and rear suspensions, respectively. $k_{s}$ and $c_{s}$ are the rigidity coefficient and damping coefficient of the chair, respectively. $k_{f}, k_{r}$ are the rigidity coefficient of front and rear suspensions, respectively. $k_{t f}, k_{t r}$ are the rigidity coefficient of front and rear wheels, respectively. $q_{f}, q_{r}$ are the road excitation forces at front and rear wheels, respectively. $l_{1}$ is the distance between chair and vehicle mass center. $l_{2}, l_{3}$ are the distances from the vehicle mass center to front and rear wheel axles, respectively. 
By Lagrange equations, the mathematical model of the vehicle mechanical model in Figure 2 is as follows:

$$
[M]\{\ddot{Z}\}+[C]\{\dot{Z}\}+[K]\{Z\}=[F]\{Q\}
$$

where

$$
\begin{aligned}
& M=\left[\begin{array}{ccccc}
m_{s} & 0 & 0 & 0 & 0 \\
0 & m_{b} & 0 & 0 & 0 \\
0 & 0 & m_{p} & 0 & 0 \\
0 & 0 & 0 & m_{f} & 0 \\
0 & 0 & 0 & 0 & m_{r}
\end{array}\right] \\
& C=\left[\begin{array}{ccccc}
c_{s} & -c_{s} & c_{s} l_{1} & 0 & 0 \\
-c_{s} & c_{s}+c_{f}+c_{r} & -c_{s} l_{1}-c_{f} l_{2}+c_{r} l_{3} & -c_{f} & -c_{r} \\
c_{s} l_{1} & -c_{s} l_{1}-c_{f} l_{2}+C r l_{3} & c_{s} l_{1}^{2}+c_{f} l_{2}^{2}+c_{r} l_{3}^{2} & c_{f} l_{2} & -C r l_{3} \\
0 & -c_{f} & c_{f} l_{1} & c_{f} & 0 \\
0 & -c_{r} & -c_{r} l_{3} & 0 & c_{r}
\end{array}\right] \\
& K=\left[\begin{array}{ccccc}
k_{s} & -k_{s} & k_{s} l_{1} & 0 & 0 \\
-k_{s} & k_{s}+k_{f}+k_{r} & -k_{s} l_{1}-k_{f} l_{2}+k_{r} l_{3} & -k_{f} & -k_{r} \\
k_{s} & -k_{s} l_{1}-k_{f} l_{2}+k_{r} l_{3} & k_{s} l_{1}^{2}+k_{f} l_{2}^{2}+k_{r} l_{3}^{2} & k_{f} l_{2} & -k_{r} l_{3} \\
0 & -k_{f} & k_{f} l_{2} & k_{f}+k_{t f} & 0 \\
0 & -k_{r} & -k_{r} l_{3} & 0 & k_{r}+k_{t r}
\end{array}\right] \text {, } \\
& F=\left[\begin{array}{cc}
0 & 0 \\
0 & 0 \\
0 & 0 \\
k_{t f} & 0 \\
0 & k_{t r}
\end{array}\right], \quad\{Z\}=\left\{\begin{array}{lllll}
z_{s} & z_{b} & z_{p} & z_{f} & z_{r}
\end{array}\right\}^{T}, \quad\{Q\}=\left\{\begin{array}{ll}
q_{f} & q_{r}
\end{array}\right\}^{T} .
\end{aligned}
$$

\subsection{Road Excitation}

The unevenness degree of road profile can be generally described by power spectrum density. The international GB7031 recommends that the power spectrum density of road profile is described by

$$
S_{q}(n)=S_{q}\left(n_{0}\right)\left(\frac{n}{n_{0}}\right)^{-w}
$$


where $S_{q}\left(n_{0}\right)$ is the unevenness coefficient of road profile. $n_{0}$ is the referenced spatial frequency, $n_{0}=0.1\left(\mathrm{~m}^{-1}\right) . n$ is the spatial frequency $\left(\mathrm{m}^{-1}\right) . w$ is the frequency exponent of the graded road spectrum and generally chosen as 2 . In this study, $S_{q}\left(n_{0}\right)$ is set as $S_{q}\left(n_{0}\right)=64 \times 10^{-6}\left(\mathrm{~m}^{2} / \mathrm{m}^{-1}\right)$, that is, Grade B road condition.

When automobiles move in changeable speeds, the excitations of automobile systems are different in time domain and space domain. It is not stationary in space domain but in time domain. However, the automobile's mechanical responses are all nonstationary. By the inherent characteristics of frequency response function $H(w)$ of vehicle system in time domain and the relation of time frequency $w$ and space frequency $n$, the transient frequency response function $H(s, n)$ can be obtained, then we can solve the stochastic vibration of the vehicle system in changeable speed moving [11,23].

The unevenness degree of roads in time domain is shown as follows [24]:

$$
q(t)=h_{0} e^{j w t}
$$

where $h_{0}$ is the amplitude of unevenness degree of roads. The expression of unevenness degree of roads in space domain is as follows:

$$
\begin{gathered}
q=h_{0} e^{j \Omega s}, \\
w t=\Omega v
\end{gathered}
$$

where $\Omega$ is the spatial angular frequency.

When the automobile is moving in a constant speed, $s=v t$, it has the following relation, $w=\Omega s$ or $f=n v$.

When the automobile is moving in a changeable speed, it has

$$
s=v_{0}+\frac{a t^{2}}{2}
$$

where $v_{0}$ is the initial velocity of the automobile and $a$ is its acceleration. Then (3.6) can be rewritten as

$$
\begin{gathered}
w d t=\Omega d s \\
w=\Omega \frac{d s}{d t}=2 n \pi\left(v_{0}+a t\right)=2 n \pi\left(2 a s+v_{0}^{2}\right)^{1 / 2} .
\end{gathered}
$$

Equation (3.8) reflects the time-space frequency relation of automobiles in an accelerated moving.

\subsection{Pseudoexcitation of Random Road at Front and Rear Wheels}

By the time-frequency expression of unevenness degree of roads, a pseudoexcitation of road $\tilde{q}(t)$ can be built which is corresponding with the road excitation $q(t)$, as follows:

$$
\tilde{q}(t)=\sqrt{S_{q}(n)} e^{i w t}
$$


Hypothesize that the road excitations on four wheels of the automobile are the same and the delay relation [14] between front wheel excitation $q_{f}(t)$ and rear wheel excitation $q_{r}(t)$ is as follows:

$$
q_{r}(t)=q_{f}(t-\tau)
$$

where $\tau=l / v, l$ is the distance between two wheel axles, then it has

$$
\begin{gathered}
\tilde{q}_{f}(t)=\sqrt{S_{q}(n)} e^{i w t}, \\
\tilde{q}_{r}(t)=\tilde{q}_{f}(t-\tau)=\sqrt{S_{q}(n)} e^{i w(t-\tau)}=e^{-i w t \tau} \tilde{q}(t) .
\end{gathered}
$$

Therefore, the excitation input is written as follows:

$$
\{\tilde{q}(t)\}=\left[\begin{array}{c}
\tilde{q}_{f}(t) \\
\tilde{q}_{r}(t)
\end{array}\right]=\left[\begin{array}{c}
1 \\
e^{-i w \tau}
\end{array}\right] \tilde{q}(t)=\left\{H_{q}(w)\right\} \tilde{q}(t) .
$$

The road excitations from front wheel and rear wheel can be simplified as an excitation input $\{\tilde{q}(t)\}$, and its frequency response characteristic is $\left\{H_{q}(w)\right\}$. Thus, the two-point excitations are simplified as one-point excitation.

\subsection{Formulation of System Response}

For a multiple degrees-of-freedom system, its frequency response characteristic is the complex number ratio of response vector and excitation vector. For the half-car, five-DOF vehicle system in this study, if we supposed that its frequency response is $[H(w)]$, then the relation of pseudoresponse and pseudoexcitation is

$$
\{\tilde{z}(t)\}=[H(w)]\{\tilde{q}(t)\}
$$

Substituting (3.12) into (3.13) gives

$$
\{\tilde{z}(t)\}=[H(w)]\left\{H_{q}(w)\right\} \tilde{q}(t)=\left\{h_{g}(w)\right\} \tilde{q}(t) .
$$

Since $\left\{h_{g}(w)\right\}=[H(w)]\left\{H_{q}(w)\right\}$, then

$$
\begin{gathered}
\{\dot{\tilde{z}}(t)\}=\left\{h_{g}(w)\right\} \dot{\tilde{q}}(t)=i w\left\{h_{g}(w)\right\} \tilde{q}(t), \\
\{\ddot{\widetilde{z}}(t)\}=-w^{2}\left\{h_{g}(w)\right\} \tilde{q}(t) .
\end{gathered}
$$

Substitute (3.15) and (3.16) into the system equation, then the system frequency response function can be obtained as follows:

$$
[H(w)]=\left[[K]-w^{2}[M]+i w[C]\right]^{-1} F,
$$


where

$$
[H(w)]_{5 \times 2}=\left[\begin{array}{c}
H(w)_{s} \\
H(w)_{b} \\
H(w)_{p} \\
H(w)_{f} \\
H(w)_{r}
\end{array}\right]
$$

The frequency response functions of the relative displacement of suspension and the dynamic loads of tires are as follows, respectively:

$$
\begin{gathered}
H_{r d}(w)=\frac{z_{b}(w)-l_{2} z_{p}(w)-z_{f}(w)}{q_{f}(w)}, \\
H_{t f}(w)=\frac{z_{f}(w)-z_{r}(w)}{q_{f}(w)} k_{t f} .
\end{gathered}
$$

By substituting (3.13) into (3.19), it gives the frequency response functions of the relative displacement of suspension and the dynamic loads of tires as follows:

$$
\begin{gathered}
H_{r d}(w)=H_{b}(w)-l_{2} H_{p}(w)-H_{f}(w), \\
H_{t f}(w)=\left[H_{f}(w)-H_{r}(w)\right] k_{t f} .
\end{gathered}
$$

It can be found that the system response result by pseudoexcitation method is the same as the result obtained by Fourier transform analysis. After obtaining the automobile structural parameters and the road excitation parameters, the pseudoexcitation responses $\{\tilde{z}(t)\}$ and $\{\ddot{z}(t)\}$ can be solved in accordance with (3.14) and (3.16). The response power spectrum can be achieved according to (2.5) and (2.6).

The power spectrum matrix of vertical acceleration of the system is

$$
\begin{aligned}
\left\{S_{\ddot{z} \ddot{z}}(w)\right\} & =\{\ddot{\tilde{z}}(t)\}^{*} \cdot\{\ddot{\tilde{z}}(t)\}^{T}=w^{4}\left\{h_{g}(w)\right\} \sqrt{S_{q}(n)} e^{-i w t}\left\{h_{g}(w)\right\}^{T} \sqrt{S_{q}(n)} e^{i w t} \\
& =w^{4}\left\{h_{g}(w)\right\}\left\{h_{g}(w)\right\}^{T} S_{q}(n) \\
& =w^{4}[H(w)]\left\{H_{q}(w)\right\}\left\{H_{q}(w)\right\}^{T}[H(w)]^{T} S_{q}(n) .
\end{aligned}
$$

By substituting $w=2 n \pi\left(2 a s+v_{0}^{2}\right)^{1 / 2}$ into (3.21), the spatial acceleration power spectrum density of system responses can be obtained as follows:

$$
\left\{S_{\ddot{z} \ddot{z}}(s, n)\right\}=(2 \pi n)^{4}\left(2 a s+v_{0}^{2}\right)^{2}[H(s, n)]\left\{H_{q}(s, n)\right\}\left\{H_{q}(s, n)\right\}^{T}[H(s, n)]^{T} S_{q}(n),
$$




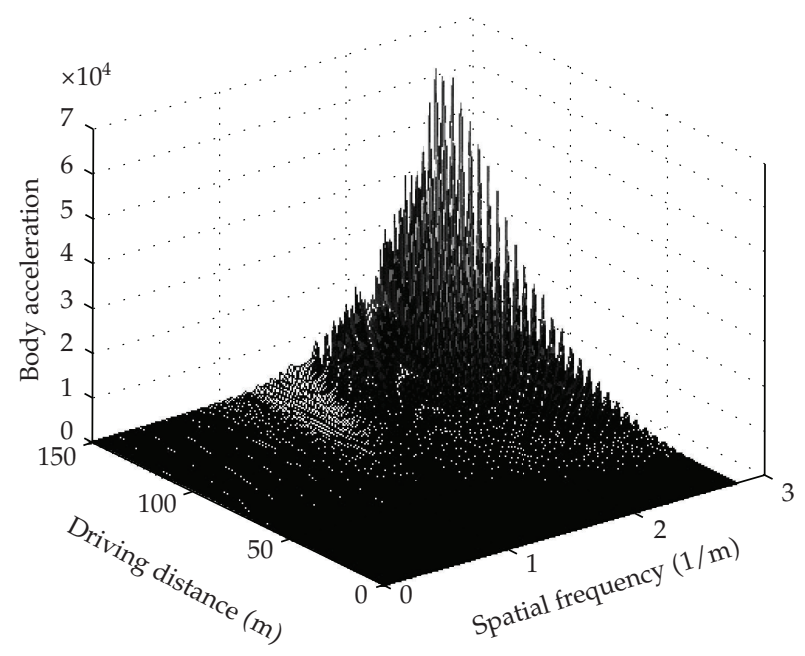

Figure 3: 3D spectrum of body acceleration.

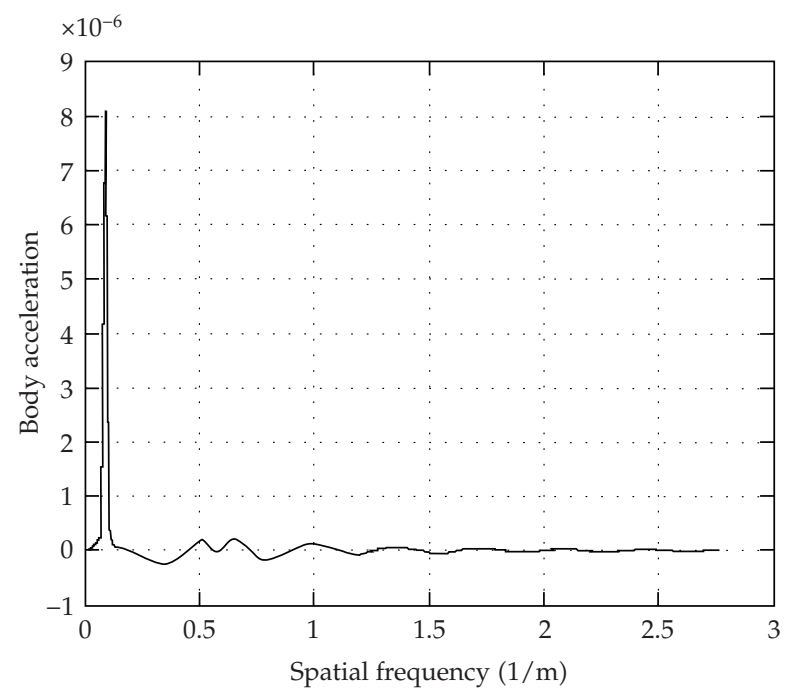

Figure 4: 2D spectrum of body acceleration.

where

$$
\begin{gathered}
H(s, n)=\left[[K]-4 n^{2} \pi^{2}\left(2 a s+v_{0}^{2}\right)[M]+i\left(2 n \pi \sqrt{2 a s+v_{0}^{2}}[C]\right)\right]^{-1} F, \\
\left\{H_{q}(s, n)\right\}=\left[\begin{array}{c}
1 \\
e^{-i\left(2 n \pi \sqrt{2 a s+v_{0}^{2}} \tau\right)}
\end{array}\right] \sqrt{S_{q}(n)} e^{-i\left(2 n \pi \sqrt{2 a s+v_{0}^{2}} t\right)} .
\end{gathered}
$$

In the same way, according to the frequency response function of relative displacement of suspensions and dynamic loads of tires, we can obtain the transient spatial power spectrum 


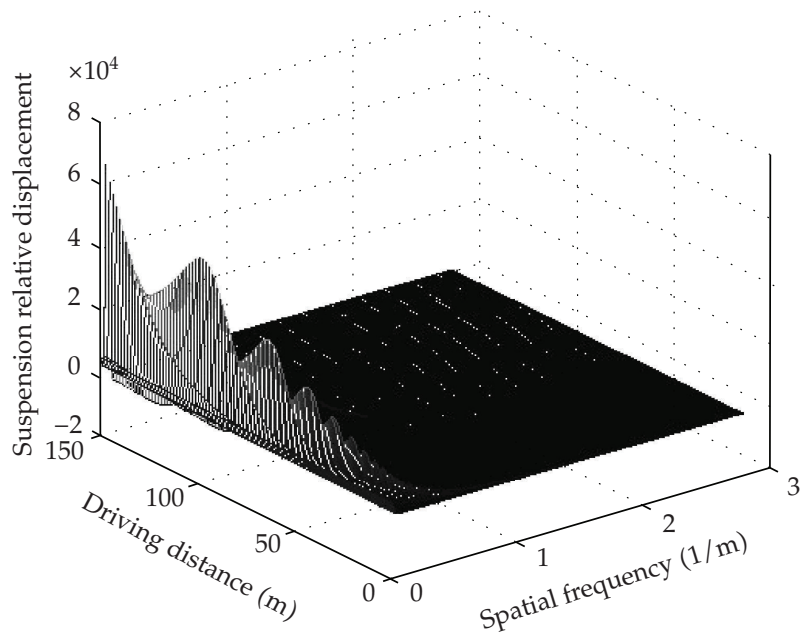

Figure 5: 3D spectrum of relative displacement of vehicle suspension.

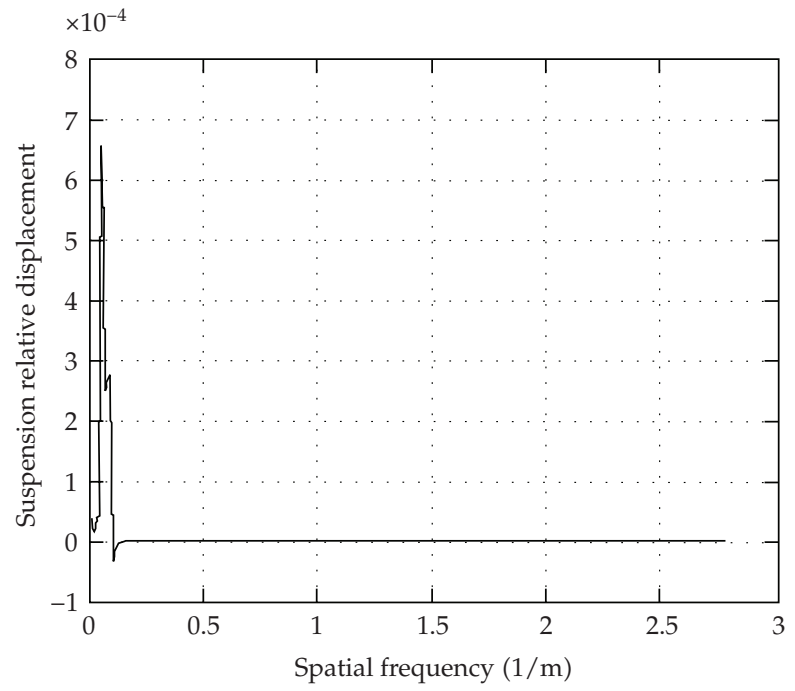

Figure 6: 2D spectrum of relative displacement of vehicle suspension.

density functions. It is important to substitute $w=2 n \pi\left(2 a s+v_{0}^{2}\right)^{1 / 2}$ into them to get the corresponding power spectrum density as follows:

$$
\begin{aligned}
& S_{r d}(s, n)=H_{r d}(s, n) S_{q}(n)\left[H_{r d}(s, n)\right]^{T}, \\
& S_{t f}(s, n)=H_{t f}(s, n) S_{q}(n)\left[H_{t f}(s, n)\right]^{T} .
\end{aligned}
$$

By means of computing $\left\{S_{\ddot{z} \ddot{z}}(s, n)\right\}, S_{r d}(s, n)$, and $S_{t f}(s, n)$, we can obtain the dynamic characteristics of vehicles and driving comfort of vehicles. 


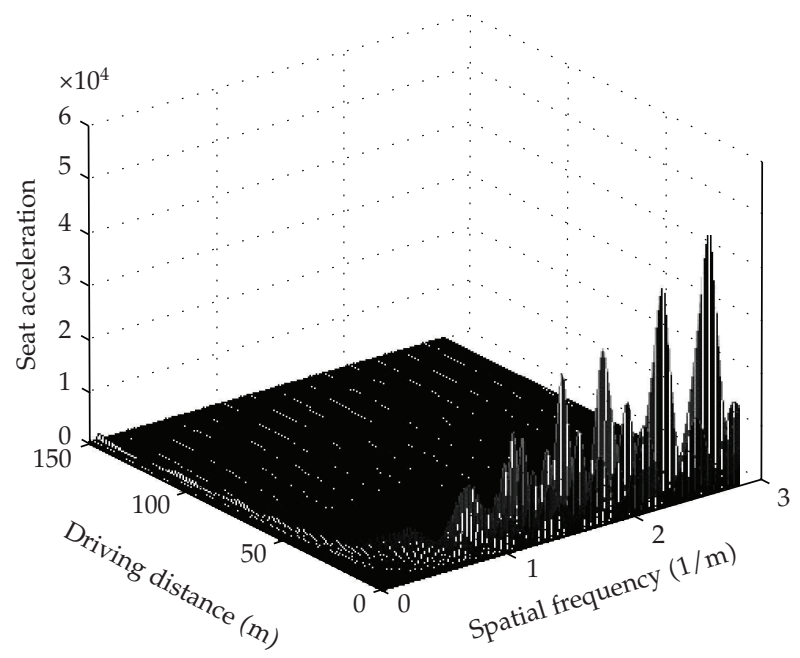

Figure 7: 3D spectrum of seat acceleration.

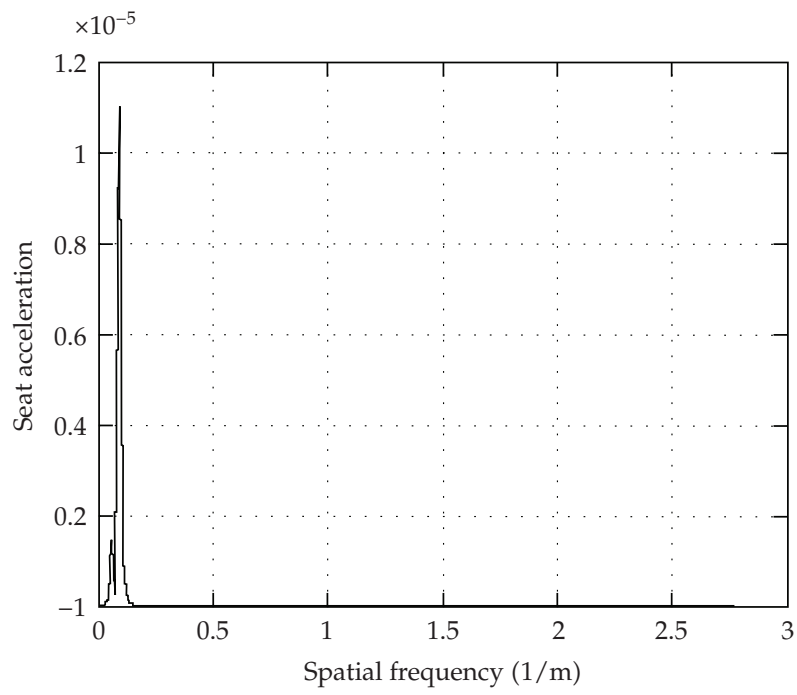

Figure 8: 2D spectrum of seat acceleration.

\section{Computing Case}

In order to check the validation of the above mathematical models of transient response analysis of the half-car, five-DOF automobile system based on pseudoexcitation, we carried out the simulations as follows. To mimic an accelerating process of the automobile, set the acceleration as $a=1.0 \mathrm{~ms}$ and the running distance as $s=150 \mathrm{~m}$, respectively. The other mechanical model parameters of the five-DOF automobile system are shown in Table 1.

Figures 3 and 4 show the 3D (three-dimensional) and 2D (two-dimensional) spectrums of body acceleration, respectively. Figures 5 and 6 show the 3D and 2D spectrums of relative displacement of vehicle suspension, respectively. Figures 7 and 8 show the 3D and 2D spectrums of seat acceleration, respectively. Figures 9 and 10 show the 3D and 


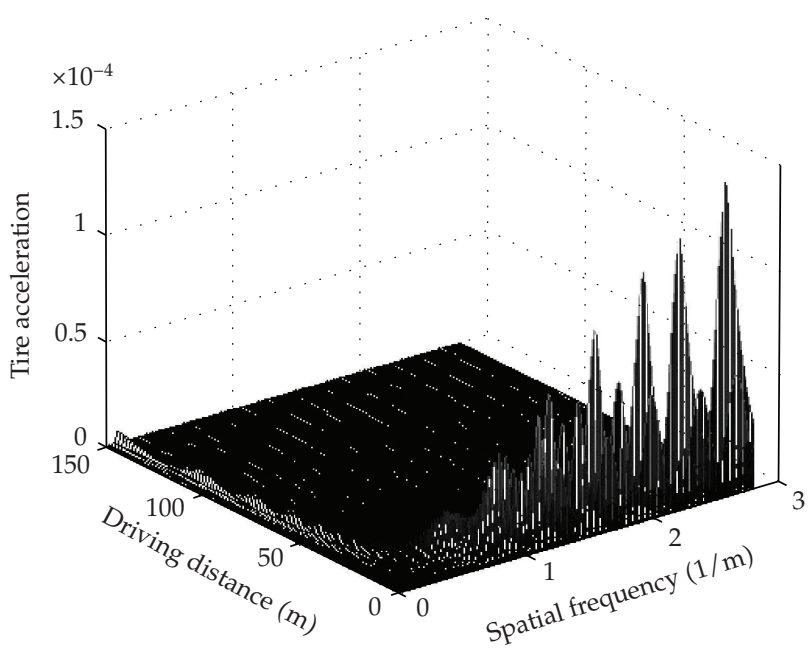

Figure 9: 3D spectrum of tire acceleration.

Table 1: Mechanical model parameters of the five-DOF automobile system.

\begin{tabular}{lccccc}
\hline Items & $m_{s}(\mathrm{~kg})$ & $m_{b}(\mathrm{~kg})$ & $m_{p}\left(\mathrm{~kg} \cdot \mathrm{m}^{2}\right)$ & $m_{f}(\mathrm{~kg})$ & $m_{r}(\mathrm{~kg})$ \\
\hline Values & 70 & 2100 & 3500 & 140 & 210 \\
Items & $k_{s}$ & $k_{f}$ & $k_{r}$ & $k_{t f}$ & $k_{t r}$ \\
Values & 12200 & 74000 & 120000 & 520000 & 520000 \\
Items & $c_{s}$ & $c_{f}$ & $c_{r}$ & & \\
Values & 550 & 1800 & 1200 & & \\
\hline
\end{tabular}

$2 \mathrm{D}$ spectrums of tire acceleration, respectively. Figure 11 shows the $2 \mathrm{D}$ spectrum of tire dynamic load. The nonstationary response spectrum analysis of the vehicle system shows that the low-spatial frequency ingredient has a main role with vehicle speed increasing when the vehicle moves in a constant acceleration. With the speed increasing, the peak values of power spectrum of seat and body acceleration, as well as the low frequency values of power spectrum of dynamic tire loads, are not monotonously increasing, and some local values are decreasing. The changing of relative displacement of vehicle suspensions is not large. The nonlinearity of the vehicle suspension is not included in the current study, this is the limitation of this vehicle dynamic model, and it will be considered in the coming study.

\section{Conclusion}

By pseudoexcitation method, the vibration response characteristics of the half-car, five-DOF automobile system were obtained. The results show that the pseudoexcitation method is more convenient than the traditional method and effectively solves the smoothness computation of vehicles while the pseudoexcitation method is used to analyze vehicle vibration under nonstationary random vibration. 


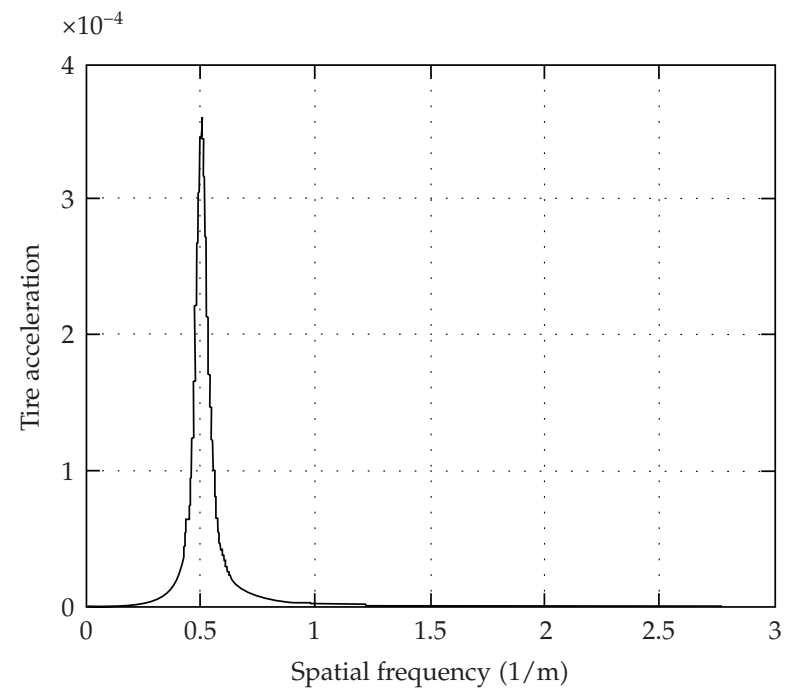

Figure 10: 2D spectrum of tire acceleration.

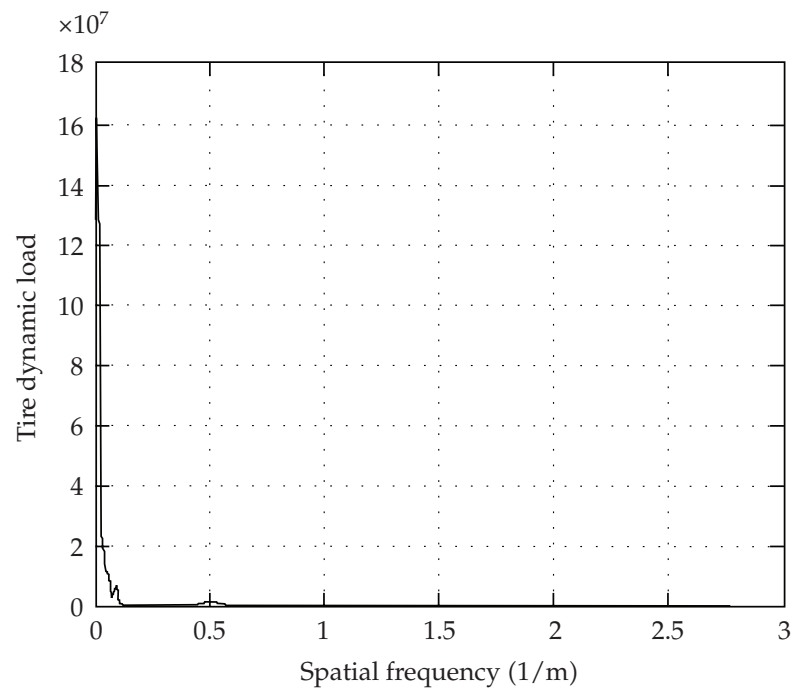

Figure 11: 2D spectrum of tire dynamic load.

\section{Acknowledgments}

The authors are grateful for the research Grants from the Program for New Century Excellent Talents in University (no. NCET-08-0103), the Scientific Research Foundation of China Postdoctor (nos. 20070420203 and 200801391), the Natural Science Foundation of China (no. 50875041), National 973 Program (2007CB210305-2), Basic Research Expense of National Universities (no. N090503001), Program for Changjiang Scholars and Innovative Research Team in University, and a research fund from Northeastern University, China. 


\section{References}

[1] J.-D. Wu and R.-J. Chen, "Application of an active controller for reducing small-amplitude vertical vibration in a vehicle seat," Journal of Sound and Vibration, vol. 274, no. 3-5, pp. 939-951, 2004.

[2] Z. S. Yu, Automobile Theory, Mechanical Industry Press, Beijing, China, 1992.

[3] P. Cacciola, N. Maugeri, and G. Muscolino, "A modal correction method for non-stationary random vibrations of linear systems," Probabilistic Engineering Mechanics, vol. 22, no. 2, pp. 170-180, 2007.

[4] H. E. M. Hunt, "Stochastic modelling of traffic-induced ground vibration," Journal of Sound and Vibration, vol. 144, no. 1, pp. 53-70, 1991.

[5] W. Gao, N. Zhang, and J. Dai, "A stochastic quarter-car model for dynamic analysis of vehicles with uncertain parameters," Vehicle System Dynamics, vol. 46, no. 12, pp. 1159-1169, 2008.

[6] A. M. A. Soliman, "Effect of road roughness on the vehicle ride comfort and rolling resistance," Tech. Rep. SAE paper no. 2006-01-1297, 2006.

[7] E. M. ElBeheiry, "Effects of small travel speed variations on active vibration control in modern vehicles," Journal of Sound and Vibration, vol. 232, no. 5, pp. 857-875, 2000.

[8] V. Rouillard and M. A. Sek, "Simulation of non-stationary vehicle vibrations," Proceedings of the Institution of Mechanical Engineers. Part D. Journal of Automobile Engineering, vol. 215, no. 10, pp. 10691075, 2001.

[9] J. Lin, W. Zhang, and J. Li, "Structural responses to arbitrarily coherent stationary random excitations," Computers and Structures, vol. 50, no. 5, pp. 629-633, 1994.

[10] F. Lu, Q. Gao, J. H. Lin, and F. W. Williams, "Non-stationary random ground vibration due to loads moving along a railway track," Journal of Sound and Vibration, vol. 298, no. 1-2, pp. 30-42, 2006.

[11] F. Lu, J. H. Lin, D. Kennedy, and F. W. Williams, "An algorithm to study non-stationary random vibrations of vehicle-bridge systems," Computers and Structures, vol. 87, no. 3-4, pp. 177-185, 2009.

[12] C. Papalukopoulos and S. Natsiavas, "Nonlinear biodynamics of passengers coupled with quarter car models," Journal of Sound and Vibration, vol. 304, no. 1-2, pp. 50-71, 2007.

[13] S. S. Parthasarathy and Y. G. Srinivasa, "Design of an active suspension system for a quarter-car road vehicle model using model reference control," Proceedings of the Institution of Mechanical Engineers. Part I. Journal of Systems and Control Engineering, vol. 220, no. 2, pp. 91-108, 2006.

[14] J. Li, Y. Y. Qin, Q. Zhao, and W. Zhang, "A pseudo excitation method for automotive vibration analysis," Mechanical Science and Technology, vol. 28, no. 3, pp. 281-285, 2009.

[15] X. Peng, X.-H. Liu, and G.-L. Wen, "Vibration analysis of vehicle at uneven speed with pseudoexcitation method," Journal of Hunan University Natural Sciences, vol. 34, no. 11, pp. 37-41, 2007.

[16] G. Litak, M. Borowiec, M. I. Friswell, and K. Szabelski, "Chaotic vibration of a quarter-car model excited by the road surface profile," Communications in Nonlinear Science and Numerical Simulation, vol. 13, no. 7, pp. 1373-1383, 2008.

[17] R. D. Naik and P. M. Singru, "Establishing the limiting conditions of operation of magneto-rheological fluid dampers in vehicle suspension systems," Mechanics Research Communications, vol. 36, no. 8, pp. 957-962, 2009.

[18] G. Litak, M. Borowiec, M. I. Friswell, and W. Przystupa, "Chaotic response of a quarter car model forced by a road profile with a stochastic component," Chaos, Solitons and Fractals, vol. 39, no. 5, pp. 2448-2456, 2009.

[19] J. H. Lin and Y. H. Zhang, Pseudo Excitation Method of Random Vibration, Science Press, Beijing, China, 2004.

[20] H. Y. Zhou, Development and control of an automotive smart suspension system, Ph.D. thesis, University of Toronto, Ontario, Canada, 2002.

[21] D. E. Williams and W. M. Haddad, "Active suspension control to improve vehicle ride and handling," Vehicle System Dynamics, vol. 28, no. 1, pp. 1-24, 1997.

[22] J. B. Hoyle, "Modelling the static stiffness and dynamic frequency response characteristics of a leaf spring truck suspension," Proceedings of the Institution of Mechanical Engineers. Part D. Journal of Automobile Engineering, vol. 218, no. 3, pp. 259-278, 2004.

[23] M. Mitschke, Vehicle Dynamics, China Communications Press, Beijing, China, 1994.

[24] L. Sun and B. S. Greenberg, "Dynamic response of linear systems to moving stochastic sources," Journal of Sound and Vibration, vol. 229, no. 4, pp. 957-972, 2000. 


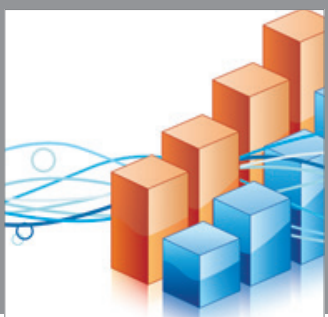

Advances in

Operations Research

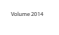

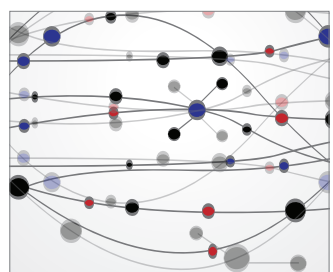

\section{The Scientific} World Journal
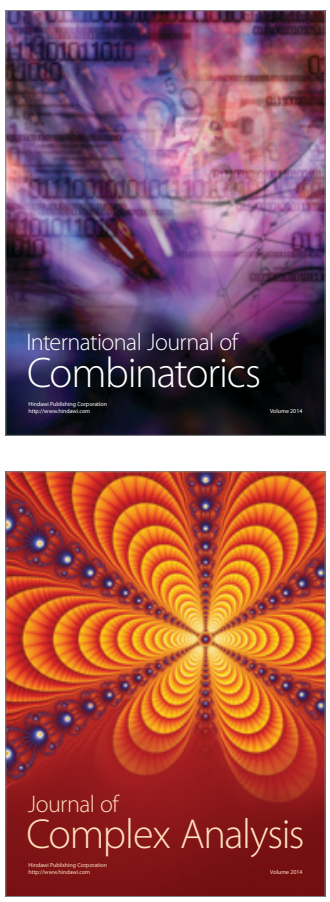

International Journal of

Mathematics and

Mathematical

Sciences
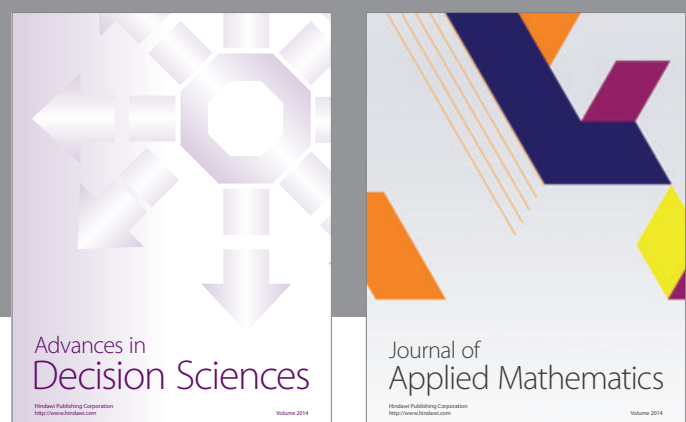

Journal of

Applied Mathematics
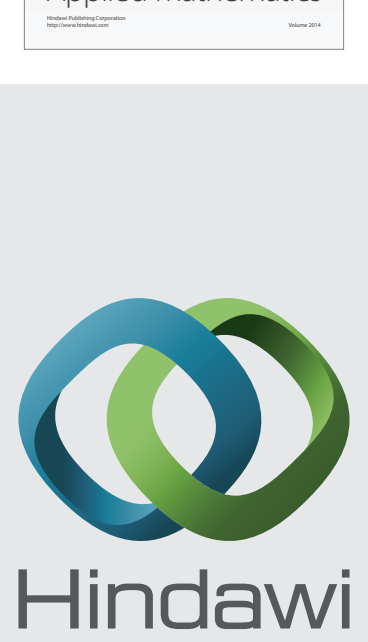

Submit your manuscripts at http://www.hindawi.com
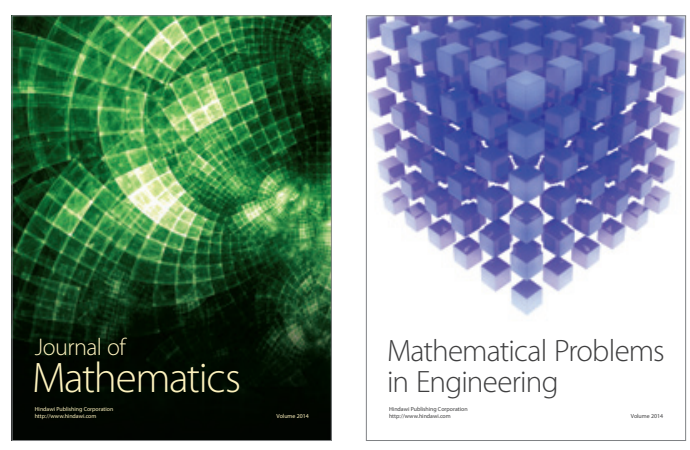

Mathematical Problems in Engineering
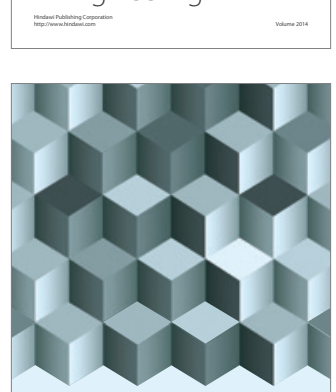

Journal of

Function Spaces
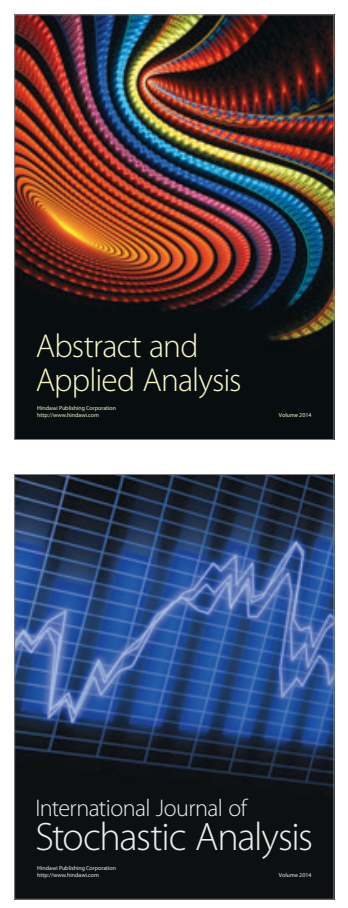

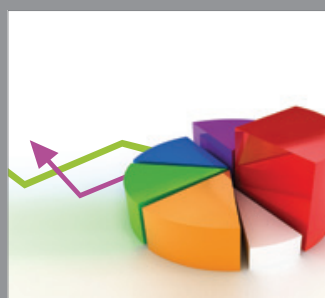

ournal of

Probability and Statistics

Promensencen
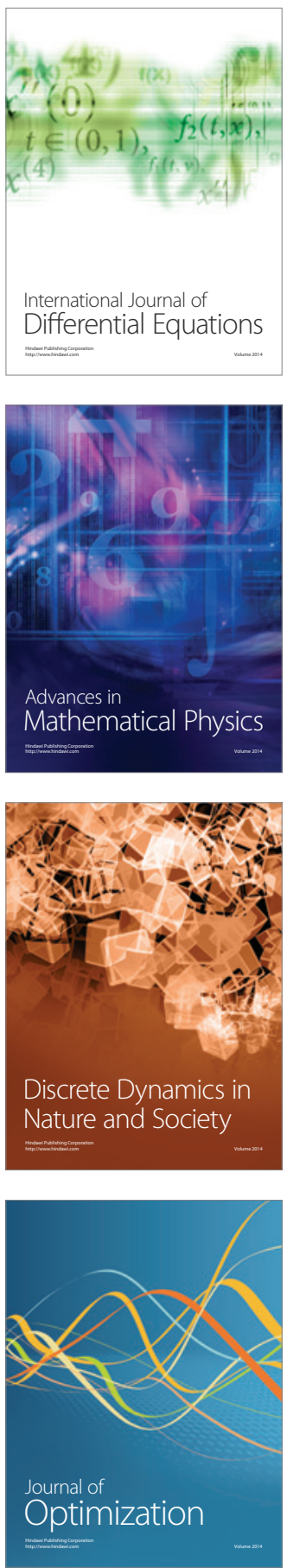